\title{
Influence of Cr doping on optical and photoluminescent properties of CdTe
}

\author{
M.I. Ilashchuk ${ }^{1}$, O.A. Parfenyuk ${ }^{1}$, K.S. Ulyanytskiy ${ }^{1}$, V.V. Brus ${ }^{1}$, N.D. Vakhnyak $^{2}$ \\ ${ }^{1}$ Yuri Fedkovych Chernivtsi National University, \\ 2, Kotsyubynsky str., 58012 Chernivtsi, Ukraine, \\ Phone: +38 (03722) 46-877,fax:+38 (03722) 46-877; e-mail: semicon@chnu.cv.ua \\ ${ }^{2} V$. Lashkaryov Institute of Semiconductor Physics, NAS of Ukraine, \\ 41, prospect Nauky,03028Kyiv,Ukraine; e-mail: div47@isp.kiev.ua
}

\begin{abstract}
Spectra of transmission and low-temperature photoluminescence of CdTe:Cr crystals have been investigated for concentrations of the doping impurity ( $\mathrm{Cr}$ ) from $1 \cdot 10^{17}$ to $4 \cdot 10^{19} \mathrm{~cm}^{-3}$ in the melt. We have found additional absorption bands with maxima at $\lambda_{1} \approx 1.9 \mu \mathrm{m}$ and $\lambda_{2} \approx 7.0 \mu \mathrm{m}$ induced by the presence of this dopant. An additional band of radiative recombination in the vicinity of $1.22 \mathrm{eV}$ is caused by electron transitions from the conduction band to the deep donor levels $E_{v}+(0.36-0.38) \mathrm{eV}$, which correspond to the $\mathrm{Cr}^{1+}$ defect entering to clusters. We have also observed the shift of CdTe:Cr absorption edge to the longwave region. This shift is caused by strong lattice deformation near the $\mathrm{Cr}^{2+}$ impurity position due to the static Jahn-Teller effect.
\end{abstract}

Keywords: CdTe, magnetic dopant, intra-center transitions, photoluminescence, transmission spectra.

Manuscript received 12.10.09; accepted for publication 22.10.09; published online 30.12.09.

\section{Introduction}

CdTe crystals doped with $\mathrm{Cr}$ atoms are a promising material for highly efficient lasers in the middle infrared spectral range [1] as well as a magnetic semiconductor for spintronic applications [2]. Further practical applications of this material depend on understanding the $\mathrm{Cr}$ impurity behaviour in CdTe crystal lattice and the mentioned dopant influence on the electrical, optical and magnetic properties. Investigation of these problems is essentially complicated by interaction of localized magnetic moments of electrons on the unoccupied $3 \mathrm{~d}$ shell between each others and the crystal band electrons. The latter causes splitting the magnetic impurity energy levels, if the impurity is localized in the initial material lattice sites. Transitions between splitted levels and allowed energy bands essentially complicate the experimental data analysis.

The objective of this work was to investigate the influence of technological factors (dopant concentration, location of samples along the ingot length) on $\mathrm{CdTe}: \mathrm{Cr}$ optical and photoluminescence spectra.

\section{Investigated objects and methodology}

The studied CdTe:Cr crystals were grown using the Bridgmen method at low $\mathrm{Cd}$ vapour pressure in the ampoule $\left(P_{\mathrm{Cd}} \approx 0.02 \mathrm{~atm}\right)$ with the purity of initial materials: Cd (99.9999\%) and Te (99.99999\%). The impurity $(\mathrm{Cr})$ concentration in the melt was between $C_{0}=\left(10^{17}-10^{19}\right) \mathrm{cm}^{-3}$. The obtained material had $p$ type conductivity and specific resistance $\rho=\left(10^{5}\right.$ $\left.10^{6}\right) \mathrm{Ohm} \cdot \mathrm{cm}$.

The samples for the measurements of transmission spectra were prepared from the different parts of the ingot with the $\mathrm{Cr}$ concentration $C_{0}=10^{19} \mathrm{~cm}^{-3}$. The photoluminescence spectra measurements at low temperature $(4.2 \mathrm{~K})$ were performed on freshly cleaved surfaces of the samples taken from the middle part of the ingots with various $\mathrm{Cr}$ concentrations.

The measurements of CdTe transmission spectra in the absorption edge region $(\lambda=0.8-2.2 \mu \mathrm{m})$ were carried out by means of МДР-23 monochromator. The infrared spectrophotometer ИКС-29 was used for measurements 
of transmission spectra within the infrared range 2.0 to $25 \mu \mathrm{m}$.

The photoluminescence spectra were investigated by the standard procedure employing the facility based on МДР-23 monochromator. The $40 \mathrm{~mW}$ power He-Ne laser was used for excitation. The spectral range of investigations was 7700 to $9300 \AA$.

\section{Results and discussion}

Transmission spectra. The spectral dependences of transmission coefficient for the CdTe:Cr crystals in the range of the absorption edge and in the transparent region are shown in Fig. 1a,b.

The obtained spectra analysis allowed us to determine the peculiarities related to doping impurities: the absorption edge location dependence on the sample position along the ingot and the shift of this edge to the longwave range comparing to pure $\mathrm{CdTe}$; two additional absorption bands with peaks at $\lambda_{1} \approx 1.9 \mu \mathrm{m}$ and $\lambda_{2} \approx 7.0 \mu \mathrm{m}$ which differ along the ingot.
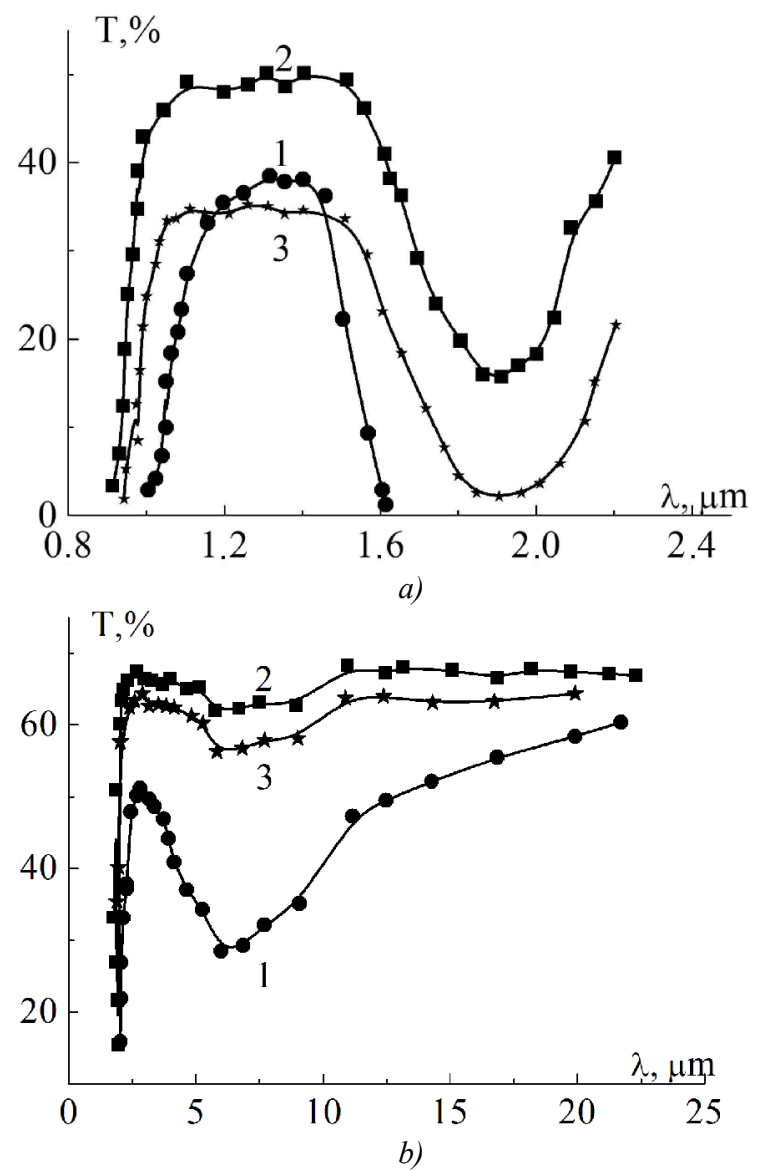

Fig. 1. Transmission spectra of CdTe:Cr crystals in the ranges of intrinsic absorption (a) and transparence (b). The dopant concentration is $C_{0}=10^{19} \mathrm{~cm}^{-3}$. Samples 1 to 3 are taken from the initial, middle, and end parts of the ingot, respectively.
The confirmation of these anomalies relationship with $\mathrm{Cr}$ impurities is their correlation with the $\mathrm{Cr}$ dopant concentration, so far as the latter depends on the samples position along the ingot.

Taking into account that the segregation coefficient of $\mathrm{Cr}$ in CdTe is less than unity [3], the mentioned peculiarities can be explained by inhomogeneous distribution of the dopant in the grown crystal. The higher $\mathrm{Cr}$ impurity concentration at the beginning of the ingot can be caused by the concentrated overcooling at the crystallization front. Thereby, the absorption edge shift to the longwave region with the increasing dopant concentration well correlates with the reported data $[4,5]$.

The observed absorption band at $\lambda_{1} \approx 1.9 \mu \mathrm{m}$, according to the literature data [6], is characteristic for $\mathrm{Cr}^{2+}$ ions in the CdTe lattice and induced by the intracenter transitions from the ground level ${ }^{5} \mathrm{~T}_{2}$ to the excited level ${ }^{5} \mathrm{E}$. Just by these terms the ground ${ }^{5} \mathrm{D}$ level of an isolated $\mathrm{Cr}^{2+}\left(3 \mathrm{~d}^{4}\right)$ ion becomes splitted in the CdTe crystalline field with the $T_{d}$-symmetry [6]. The confirmation of such explanation is the dependence of absorption band intensity on the impurity concentration.

As the intensity of the absorption band at $\lambda_{2} \approx 7.0 \mu \mathrm{m}$ is also determined by the $\mathrm{Cr}$ concentration, one can suppose that it is induced by the intra-center transitions in the $\mathrm{Cr}$ ion that is in another charge state. The possibility of impurities with unoccupied 3d-shells to be in different charge states in II-VI compounds was ascertained in the works $[7,8]$. There is the highest probability to suggest that the absorption band at $\lambda_{2} \approx 7.0 \mu \mathrm{m}$ corresponds to the intra-center transitions ${ }^{4} \mathrm{~T}_{1}(\mathrm{~F}) \Rightarrow{ }^{4} \mathrm{~T}_{2}(\mathrm{~F})$ in $\mathrm{Cr}^{3+}$ ion.

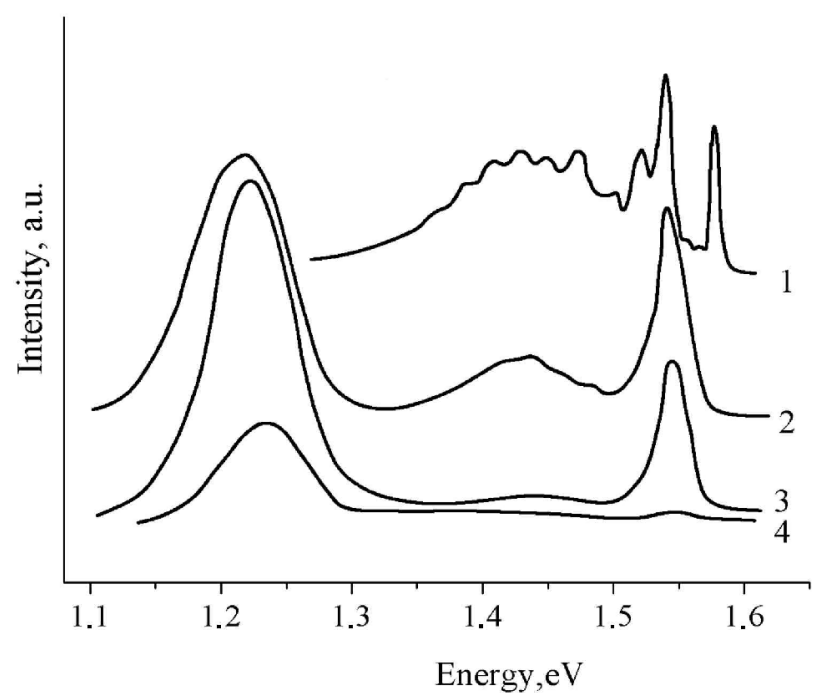

Fig. 2. The photoluminescence spectra $(T=4.2 \mathrm{~K})$ of $\mathrm{CdTe}$ samples: $1-$ undoped and Cr-doped with various concentrations $C_{0}\left(\mathrm{~cm}^{-3}\right): 2-2 \cdot 10^{17}, 3-10^{18}, 4-4 \cdot 10^{19}$. 
Photoluminescence spectra. To fuller investigate the $\mathrm{Cr}$ impurity influence on the CdTe optical properties, we studied also CdTe:Cr crystal photoluminescence spectra along with those for pure CdTe material obtained under the same technological conditions.

It is possible to separate some groups of lines inherent to the pure CdTe crystal photoluminescence spectrum (Fig. 2, curve 1). In accord with the literature data [9], the shortest wavelength band with its peak at $1.59 \mathrm{eV}$ is related with annihilation of the exciton bounded to the shallow acceptor $\left(E_{A 1}=E_{v}+0.05 \mathrm{eV}\right)$. The intensive band with the energy $1.547 \mathrm{eV}$ that corresponds to the phononless transition of edge luminescence is formed due to electron transition from the conduction band to the shallow acceptor [9]. The energy location of noticed acceptor level, taking into account the value $E_{g}=1.606 \mathrm{eV}$ at $4.2 \mathrm{~K}$, is determined as $E_{A 1}=E_{v}+0.059 \mathrm{eV}$. The determined value $E_{A 1}$ is very close to the acceptor location $E_{v}+0.05 \mathrm{eV}$, which is created in the CdTe material that was grown at a low $\mathrm{Cd}$ vapour pressure and is related to the isolated singly ionized $\mathrm{Cd}$ vacancy or to the complex with its participation [9]. The closest two longwave bands are the LO-phonon recurrence of the noticed band (for the CdTe $h v_{\mathrm{LO}}=0.021 \mathrm{eV}$ ). The wide photoluminescence region at $1.42 \mathrm{eV}$ is typical for CdTe [9]. Beside the electron transition, it is characterized by a number of emission peaks related to the various quantity of optic LO phonons. This band can be explained by the transition to the acceptor centers with the energy location $E_{A 2}=E_{v}+(0.12-0.18) \mathrm{eV}$. These defects are considered to have a complex nature, and they consist of shallow acceptors and doubly ionized $\mathrm{Cd}$ vacancies (Acenters) [10]. It is the most probable to suggest that the A-centers in the investigated crystals are created by the influence of background $p$-type impurities.

Significant changes in the CdTe luminescent spectrum are observed at a relatively low concentration of impurities $\left(C_{0}=2 \cdot 10^{17} \mathrm{~cm}^{-3}\right)$. Injection of $\mathrm{Cr}$ atoms into CdTe lattice causes entire disappearance of exciton luminescence, strong quenching the edge luminescence at $1.42 \mathrm{eV}$ as well as the appearance of additional luminescence in the region close to $1.22 \mathrm{eV}$. At the highest $\mathrm{Cr}$ concentration $\left(C_{0}=4 \cdot 10^{19} \mathrm{~cm}^{-3}\right)$ the luminescence spectrum practically consists of one emission band with the peak close to $1.22 \mathrm{eV}$. As far as the acceptors $\mathrm{A}_{1}\left(E_{v}+0.05 \mathrm{eV}\right)$ and $\mathrm{A}_{2}\left(E_{v}+(0.12-0.18) \mathrm{eV}\right)$ can be interpreted as $\mathrm{V}_{\mathrm{Cd}}^{\prime}$ and $\left(\mathrm{V}_{\mathrm{Cd}}^{\prime \prime} \mathrm{D}^{\prime}\right)^{\prime}[9,10]$, the observed quenching of the appropriate luminescence bands at $\mathrm{Cr}$ concentration increase is due to the decrease of $\mathrm{V}_{\mathrm{Cd}}$ vacancies density because of dilution of $\mathrm{Cr}$ atoms in the cadmium sublattice. It is necessary to underline that the donor-acceptor couples radiation quenching takes place because of injection of the Fe impurity [11] as well as the elements of the IV group $\mathrm{Ge}, \mathrm{Sn}, \mathrm{Pb}$ $[12,13]$ into the CdTe lattice.
A feature of the additional emission band in photoluminescence spectra of $\mathrm{CdTe}: \mathrm{Cr}$ crystals is the dependence of the peak position on the dopant concentration. Its shift to short wavelengths with increasing the $\mathrm{Cr}$ impurity concentration from $1.225 \mathrm{eV}$ for $C_{0}=2 \cdot 10^{17} \mathrm{~cm}^{-3}$ to $1.247 \mathrm{eV}$ for $C_{0}=4 \cdot 10^{19} \mathrm{~cm}^{-3}$ is observed. It indicates a complex nature of $\mathrm{Cr}$-induced defects, the transitions to which is the reason of origin of the mentioned PL. The obtained results are well correlated with the reported results [5]. The authors of this work had determined the existence of deep level for the CdTe:Cr crystals with different impurity concentration, responsible for the equilibrium conductivity within the energy range $E_{v}+(0.19-0.32) \mathrm{eV}$. These centers are related to the $\mathrm{Cr}$ impurities in $\mathrm{Cr}^{+1}$ charge state, which act as the deep donors in the case of impurity clusterization. Taking into account the energy gap $E_{g}$ value, the described photoluminescence band registered by us in CdTe:Cr crystals within the energy range (1.225-1.247) eV can be explained by electron transitions from the conduction band to the energy levels $E_{v}+(0.36-0.38) \mathrm{eV}$, which is in good agreement with the ionization energy value $E_{v}+0.32 \mathrm{eV}$ reported in that work.

The absence of exciton luminescence and absorption edge shift to the longwave region after injection of $\mathrm{Cr}$ atoms into CdTe lattice observed in this work can be related to the specific impurity properties. It is necessary to underline that the questions concerning the reasons of the absorption edge shift in the CdTe:Cr crystals and dependence of its value on the impurities concentration have been discussed for a long time. It is usually considered that the reason is strong lattice deformation characteristic for the $\mathrm{Cr}^{2+}$ ions in CdTe near the impurity location because of the static Jahn-Teller effect [6]. In addition, the $\mathrm{Cd}^{2+}$ ion substitution by $\mathrm{Cr}^{2+}$ one can cause lattice deformation due to the difference in ion radii ( 0.96 and $0.83 \AA$, respectively). This local lattice deformation, at a definite dopant concentration, can cause destruction of the exciton states and also induce an additional absorption near the absorption edge [4].

\section{References}

1. A.G. Bluiett, U. Hommerich, R.T. Shah, S.B. Trsvedi, S.W. Kutcher, C.C. Wang, Observation of lasing from $\mathrm{Cr}^{2+}$ :CdTe and compositional effects in $\mathrm{Cr}^{2+}$-doped II-VI semiconductors // J. Electronic Mater. 31(7), p. $806-810$ (2002).

2. K.Y. Ko, M.G. Blamire, Temperature dependent magnetization in Cr-doped CdTe crystals // Appl. Phys. Lett. 88, 172101-172104 (2006).

3. L. Kuchař, J. Drápala, J. Luňáček, Purification methods of $\mathrm{Cd}$, Te and $\mathrm{CdTe}$ periodicity of segregation coefficients of admixtures // J. Cryst. Growth 161, p. 94-103 (1996). 
4. P.I. Babiy, N.P. Gavaleshko, Yu.P. Gnatenko, P.A. Skubenko, V.I. Oleinik, Optical properties of CdTe crystals doped with V and $\mathrm{Cr} / /$ Fizika tekhnika poluprov. 12 (11), p. 2202-2206 (1978), in Russian.

5. E.S. Nikonyuk, Z.I. Zakharuk, M.I. Kuchma, M.O. Kovalets, A.I. Rarenko, I.M. Yurichuk, Compensation of hole conductivity in CdTe crystals doped with $\mathrm{Cr} / /$ Semiconductor Physics, Quantum Electronics and Optoelectronics 10(3), p. 77-79 (2007).

6. J.T. Vallin, G.A. Slack, S. Roberts, Infrared absorption in some II-VI compounds doped with $\mathrm{Cr}$ // Phys. Rev. B 2(11), p. 4313-4332 (1970).

7. V.F. Agekjan, Intra-center transitions at ions of the iron group in semiconductor II-VI matrices // Fizika tverdogo tela 44(11), p. 1921-1939 (2002), in Russian.

8. V.V. Slyn'ko, P.I. Babiy, R.V. Hamernik, Yu.P. Gnatenko, A state of the dopant of nickel in cadmium telluride // Fizika tekhnika poluprov. 28(3), p. 506-509 (1994), in Russian.
9. K.R. Zanio, Cadmium telluride, in: Semiconductors and Semimetals 13, p. 235. Academic Press, New York, San Francisco, 1978.

10. B.K. Meyer, W. Stadler, D.M. Hofmann, P. Omling, D. Sinerius, K.W. Benz, On the nature of the deep $1.4 \mathrm{eV}$ emission bands in CdTe - a study with photoluminescence and ODMR spectroscopy // J. Cryst. Growth 117, p. 656-659 (1992).

11. N.P. Gavaleshko, R.D. Ivanchuk, M.V. Kurik, I.F. Skitsko, A.V. Savitskiy, The absorption edge Fe-doped CdTe // Ukrainskiy fizich. zhurnal XX (3), p. 456-458 (1975), in Russian.

12. P. Fernández, Defect structure and luminescence properties of CdTe based compounds // J. Optoelectron. and Adv. Mater. 5(2), p. 369-388 (2003).

13. A.V. Savitskiy, O.A. Parfenyuk, M.I. Ilashchuk, A.I. Savchuk, S.M. Chupyra, Eguilibrium characteristics and low-temperature photoluminescence of $\mathrm{CdTe}: \mathrm{Pb}$ single crystals // Fizika tekhnika poluprov. 38 (5), p. 516-531 (2004), in Russian. 